\title{
Similarity judgments of same-category object representations: Effects of physical size, manipulability, and word frequency
}

\section{Magda L. Dumitru \& Gitte H. Joergensen}

To cite this article: Magda L. Dumitru \& Gitte H. Joergensen (2015) Similarity judgments of same-category object representations: Effects of physical size, manipulability, and word frequency, Visual Cognition, 23:7, 855-859, DOI: 10.1080/13506285.2015.1093242

To link to this article: http://dx.doi.org/10.1080/13506285.2015.1093242

曲 Published online: 20 Oct 2015.

Submit your article to this journal $匚$

WI Article views: 7

Q View related articles $\sqsubset$

View Crossmark data $₫$ 


\title{
References
}

Chun, M. M., \& Jiang, Y. (1998). Contextual cueing: Implicit learning and memory of visual context guides spatial attention. Cognitive Psychology, 36(1), 28-71. doi:10. 1006/cogp.1998.0681

Henderson, J. M., \& Hollingworth, A. (2003). Eye movements and visual memory: Detecting changes to saccade targets in scenes. Perception \& Psychophysics, 65(1), 58-71. doi:10.3758/BF03194783

Howard, M. W., \& Kahana, M. J. (2002). A distributed representation of temporal context. Journal of Mathematical Psychology, 46(3), 269-299. doi:10.1006/jmps.2001.1388

Irwin, D. E., \& Zelinsky, G. J. (2002). Eye movements and scene perception: Memory for things observed. Perception \& Psychophysics, 64(6), 882-895. doi:10.3758/BF03196793

Noton, D., \& Stark, L. (1971). Scanpaths in saccadic eye movements while viewing and recognizing patterns. Vision Research, 11(9), 929-IN8. doi:10.1016/0042-6989(71) 90213-6

Ryan, J. D., Hannula, D. E., \& Cohen, N. J. (2007). The obligatory effects of memory on eye movements. Memory, 15(5), 508-525. doi:10.1080/09658210701391022

\section{Similarity judgments of same-category object representations: Effects of physical size, manipulability, and word frequency}

\author{
Magda L. Dumitru ${ }^{a}$ and Gitte H. Joergensen ${ }^{b}$ \\ ${ }^{a}$ Department of Cognitive Science, METU Informatics Institute, Ankara, Turkey; ${ }^{b}$ Department of \\ Psychology, University of Connecticut, Storrs, USA
}

\begin{abstract}
Studies of object similarity have focused on the relationship between different physical objects and their mental representations or between instances of the same physical object and its mental representation. The present study is the first to investigate the structure of within-category psychological space. We provided evidence that large objects and frequently mentioned objects are perceived as less similar to each other compared to small objects or less frequently mentioned objects. Further, similarity judgments were higher for manipulable objects compared to non-manipulable objects. The relevance of these data to the isomorphism between physical and psychological spaces is also discussed.
\end{abstract}

ARTICLE HISTORY Received 18 July 2015; Accepted 17 August 2015

KEYWORDS Similarity; category representation; manipulability; isomorphy of psychological and physical space 
Similarity affects categorization and recognition performance depending on changes in viewing conditions and therefore plays a central role in object representation theories. Indeed, a particular object never appears quite the same to the point that slight differences between two instances of the same object may sometimes appear greater than differences between distinct objects. According to Shepard's universal law of generalization (Shepard, 1987; also see Chater \& Vitanyi, 2003), which is one of the few general psychological laws that govern human cognition, the probability of perceiving two objects as being similar is a negative exponential function of the distance between them in an internal psychological space. A psychological space can be considered to be analogous to physical space, where the location of an object is determined by its positions on a set of dimensions. The dimensions defining an object's physical space include spatial coordinates, whereas the dimensions defining an object's psychological space might include colour, shape, or functional attributes.

Research so far has focused on the mechanisms allowing subjects to recognize a given object by comparing its properties with the properties of an internal representation held in long-term memory. Research has also focused on how the relationships between two object representations might influence the relationships observed between two physical objects. Casasanto (2008) as well as Boot and Pecher (2010), for instance, reported that spatial proximity affects people's similarity judgments of corresponding concepts such that two objects standing close to each other are perceived as being more similar than two objects standing far from each other. Nevertheless, similarity studies have never explored the structure of within-category psychological space. The present study employed language stimuli to directly tap into the relationships between same-category objects in psychological space. We hypothesized that the isomorphism between psychological and physical spaces along dimensions such as physical size, manipulability, and familiarity (e.g., word frequency) could affect similarity judgments of samecategory object representations.

In our study, participants $(N=22)$ were first presented with one of 100 plural names of non-manipulable objects (animals such as "wolves" or "doves") or of manipulable objects (plants such as "cabbages" or "peas") chosen at random. Fruit and vegetables are considered to be highly manipulable because they can be grasped and used for various purposes, whereas living things are much less manipulable even when graspable because they cannot be held in one place for any foreseeable period or time, nor can they fulfil a specific functional usage (Salmon, McMullen, \& Filliter, 2010). Participants' task was to judge the likelihood that a similarity statement about objects belonging to the same category was true. Specifically, after reading the word "wolves", for instance, they rated the statement "They can be easily mistaken for each other" on a scale from 0 ("not very likely") to 10 
A

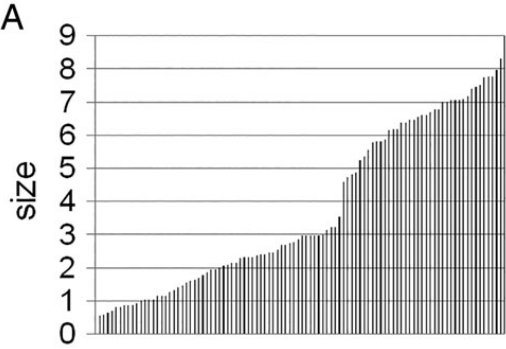

100 objects

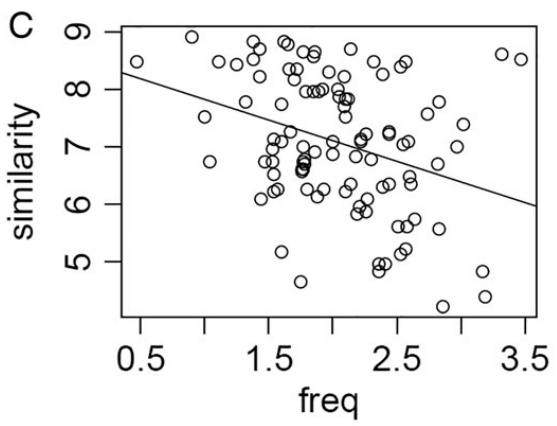

B

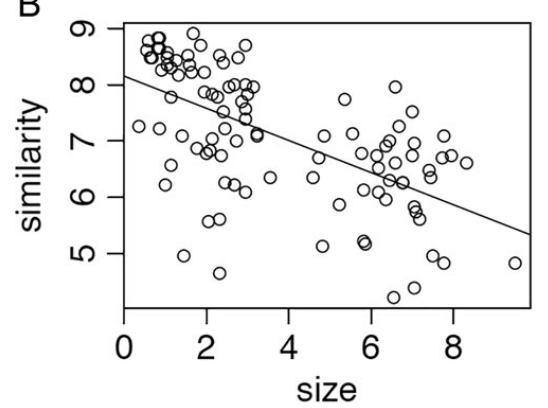

D

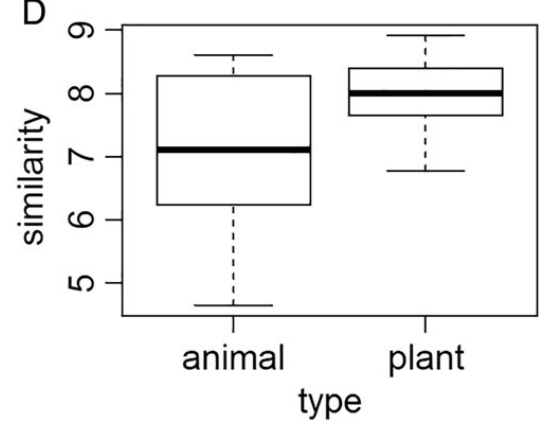

Figure 1. Size judgements on a scale from 0 ("not very big") to 10 ("very big") for 100 objects (A), similarity judgments in a scale from 0 ("not very likely" for objects to be easily mistaken for each other) to 10 ("very likely" for objects to be easily mistaken for each other) as a function of object size (B) and word frequency (C) for the 100 objects, and as a function of type that is, manipulability for 20 big objects and 20 small objects matched for word length and frequency (D).

("very likely"). Objects were of various sizes, as determined from a rating study where participants rated objects on a scale from 0 ("not very big") to 10 ("very big"). The similarity judgments we obtained are summarized in Figure 1. We fit a linear model to the data and obtained inverse effects of size and frequency on similarity ratings, adjusted $R^{2}=0.436, F(2,97)=39.41, p<.001$. We further investigated whether manipulability plays a role in similarity judgments by selecting the names of 20 animals from the list of 100 objects, which had been judged to be smallest and the names of 20 plants matching them in object size and word frequency. The $t$-test over similarity ratings for the two types was significant $(2.92, p=.005)$, indicating a direct effect of object manipulability on similarity ratings.

Taken together, our results demonstrate that large objects and frequently mentioned objects are perceived as being less similar to each other compared to small objects or less frequently mentioned objects. Interestingly, although object manipulability as well as word frequency might suggest a rather high degree of familiarity, they yielded opposite effects such that manipulable objects were perceived as being more similar to each other than non-manipulable objects. We attribute this effect to a certain tendency towards extending body ownership to those objects that people can easily manipulate, which results in a reduced ability to discriminate between inalienable objects. Indeed, as shown in Clark (2004) and in Schultz and Kuiken (2011), for 
instance, manipulable objects are often experienced as extensions of one's body, which in turn facilitate cross-modal integration in peripersonal space (Van Elk \& Blanke, 2011). Alternatively, manipulable objects are perceived as being similar to each other because they can be made to occupy a very limited space (e.g., they can be stacked up in piles).

The data confirm our hypothesis that physical and psychological spaces are isomorphic thereby allowing physical characteristics to affect the structure of psychological space. The data further allow us to draw an intriguing conclusion, namely that people usually sample a relatively equal number of objects from any given category or adjust their viewing point so as to obtain perceptual fields that are equivalent in size. Therefore, since a certain number of small objects occupies less physical space compared to the same number of large objects (e.g., a hundred ants might occupy the same floor surface as three people), humans tend to judge objects which are tightly grouped together and thus need little functional space (e.g., for bending forward, stretching their limbs, or stepping aside) as having a high degree of similarity. In contrast, large objects are perceived as being more sparsely grouped because they need considerable functional space; hence they will appear less similar to each other (cf. Shepard's law of generalization).

\section{Disclosure statement}

No potential conflict of interest was reported by the authors.

\section{Funding}

This work was supported by a Marie Curie FP7-PEOPLE-IAPP [grant number 610986].

\section{References}

Boot, I., \& Pecher, D. (2010). Similarity is closeness: Metaphorical mapping in a conceptual task. The Quarterly Journal of Experimental Psychology, 63 (5), 942-954. doi:10. 1080/17470210903134351

Casasanto, D. (2008). Similarity and proximity: When does close in space mean close in mind? Memory \& Cognition, 36 (6), 1047-1056. doi:10.3758/MC.36.6.1047

Chater, N., \& Vitanyi, P. M. B. (2003). The generalized law of generalization. Journal of Mathematical Psychology, 47, 346-369. 36. doi:10.1016/S0022-2496(03)00013-0

Clark, A. (2004). Natural-born cyborgs: Minds, technologies and the future of human intelligence. Oxford: Oxford University Press. ISBN 0-19-514866-5.

Salmon, J. P., McMullen, P. A., \& Filliter, J. H. (2010). Norms for two types of manipulability (graspability and functional usage), familiarity, and age of acquisition for 320 photographs of objects. Behavior Research Methods, 42 (1), 82-95. doi:10.3758/ BRM.42.1.82

Schultz, A. E., \& Kuiken, T. A. (2011). Neural interfaces for control of upper limb prostheses: The state of the art and future possibilities. $P M \& R, 3,55-67$. doi:10.1016/j. pmrj.2010.06.016 
Shepard, R. N., (1987). Toward a universal law of generalization for psychological science. Science, 237, 1317-1323. doi:10.1126/science.3629243

Van Elk, M. , \& Blanke, O. (2011). Manipulable objects facilitate cross-modal integration in peripersonal space. PLOS ONE, 6 (9), e24641. doi:10.1371/journal.pone.00224641

\title{
Visual long-term memory has weaker fidelity than working memory
}

\author{
M. W. Schurgin and J. I. Flombaum \\ Department of Psychological and Brain Sciences, Johns Hopkins University, Baltimore, MD, \\ USA
}

\begin{abstract}
Recent research suggests that visual long-term memory (VLTM) and visual working memory (VWM) possess similar resolution for feature memory. We investigated resolution in more holistic terms, exploiting a two alternative forced choice (2AFC) procedure and injecting noise into stimuli. Participants were exposed to two real-world objects per trial in a VWM experiment. One object was tested after a short delay. The other object (from each trial) was tested in a later session. We observed better performance for VWM, and the two systems were affected by noise in distinct ways. These results have broad implications for theories of visual memory.
\end{abstract}

\section{ARTICLE HISTORY Received 14 July 2014; Accepted 25 August 2015}

KEYWORDS Memory; Long-term memory; Working memory; Object recognition

Although visual working memory (VWM) and visual long-term memory (VLTM) are thought to be distinct systems (Broadbent, 1957; Jonides et al., 2008), they also clearly interact with and depend on one another in a variety of ways. Indeed, working memory maintenance appears to be a critical step for long-term encoding (Ranganath, Cohen, \& Brozinsky, 2005). An important set of questions concerning how they interact pertains to the nature of the representations they employ, their contents, formats, and resolution. Under the assumption that they traffic in the same representational contents and formats, recent research has investigated the resolutions of the two systems in terms of colour. If an object is seen in a particular colour, how precisely can that colour be recalled on the basis of working memory, and on the basis of long-term memory? Using a delayed estimation procedure, Brady, 